\title{
Nomogram to differentiate between aortic dissection and non- ST segment elevation acute coronary syndrome: a retrospective cohort study
}

\author{
Baowei Zhang $^{1,2 *}$, Yingying Wang ${ }^{2 \#}$, Junfang Guo ${ }^{2}$, Guohui Zhang ${ }^{2}$, Bing Yang ${ }^{1}$ \\ ${ }^{1}$ Center of Cardiology, Shanghai East Hospital, Tongji University School of Medicine, Shanghai, China; ${ }^{2}$ Department of Cardiology, the affiliated \\ People's Hospital of Jiangsu University, Zhenjiang, China \\ Contributions: (I) Conception and design: B Zhang, G Zhang, B Yang; (II) Administrative support: J Guo, G Zhang, B Yang; (III) Provision of study \\ materials or patients: J Guo, G Zhang, B Yang; (IV) Collection and assembly of data: B Zhang, Y Wang; (V) Data analysis and interpretation: B \\ Zhang, Y Wang; (VI) Manuscript writing: All authors; (VII) Final approval of manuscript: All authors. \\ "Theses authors contributed equally to this work. \\ Correspondence to: Guohui Zhang, MD. Department of Cardiology, the affiliated People's Hospital of Jiangsu University, 8 Dianli Road, Zhenjiang \\ 212002, China. Email: guohuizhang2013@126.com; Bing Yang, MD. Center of Cardiology, Shanghai East Hospital, Tongji University School of \\ Medicine, Jimo Road 150, Shanghai 200120, China. Email: ybheart@126.com.
}

Background: Aortic dissection (AD) and non-ST segment elevation acute coronary syndrome (ACS) are two of the most life-threatening diseases encountered in the emergency department (ED), but there are no rapid and reliable tools for differentiation. The purpose of this study is to develop and validate a nomogram that incorporates both the clinical characteristics and bedside laboratory tests available to differentiate between AD and non-ST segment elevation ACS (NSTE-ACS).

Methods: Between January 2016 and July 2018, patients with AD and NSTE-ACS were enrolled and divided into training and validation groups. The least absolute shrinkage and selection operator (LASSO) regression model was used to select the factors with significant value of predicting the diagnosis of AD. A nomogram was built on the basis of multivariable logistic regression analysis. Area under the curve (AUC) of receiver operating characteristic (ROC) curve and the calibration curve were used to assess the performance of the nomogram. Decision curve analysis was performed to assess the clinical utility of the nomogram.

Results: A final cohort of 263 patients (94 patients with AD and 169 patients with NSTE-ACS) were enrolled. Six variables were incorporated in the nomogram: pain severity, tearing pain, pulse asymmetry, electrocardiogram (ECG), D-dimer level and troponin I level. The AUC of the nomogram to predict the probability of $\mathrm{AD}$ was 0.919 (95\% CI, 0.876-0.962) in the training group and 0.938 (95\% CI, 0.888-0.989) in the validation group. The calibration curve demonstrated a good consistency between the actual clinical results and the predicted outcomes. The decision curve analysis indicated that the nomogram had higher overall net benefits in predicting $\mathrm{AD}$ in both the training group and the validation group.

Conclusions: We developed and validated a predictive nomogram that could be used as a tool to differentiate AD from NSTE-ACS rapidly and accurately.

Keywords: Aortic dissection (AD); non-ST segment elevation acute coronary syndrome (ACS); chest pain; D-dimer; nomogram

Submitted Nov 14, 2020. Accepted for publication Jan 18, 2021.

doi: $10.21037 / \mathrm{cdt}-20-935$

View this article at: http://dx.doi.org/10.21037/cdt-20-935 


\section{Introduction}

Aortic dissection (AD) and acute coronary syndrome (ACS) are two of the most life-threatening diseases encountered in the emergency department (ED) (1). Early and intensive anti-thrombotic therapy is crucial for patients with ACS, but it may be catastrophic for patients with $\mathrm{AD}$ (2). Therefore, differentiating AD from ACS rapidly and accurately is of great significance. However, because these two diseases share similar risk factors and clinical characteristics, a fast and accurate differential diagnosis is challenging for clinicians. For patients with ST segment elevation myocardial infarction, there are significant characteristics of electrocardiogram (ECG) for differentiating between these conditions. However, for patients with $\mathrm{AD}$ and nonST segment elevation ACS (NSTE-ACS), there are no fast and reliable tools for differentiation. Currently, some noninvasive imaging methods, such as aortic computed tomography angiography (CTA) and magnetic resonance angiography (MRA), are used to diagnose $\mathrm{AD}$ (3), but these methods are time-consuming and unavailable at bedside. Echocardiography is a bedside tool that is able to provide important signs for diagnosis of $\mathrm{AD}$, especially in patients with unstable hemodynamics, but the diagnostic accuracy and technical feasibility are limitations in ED (4).

The AD detection (ADD) risk score, which includes predisposing conditions, pain features and physical findings, is a simple assessment tool which guides the diagnostic approach for patients with chest pain (5). However, this ADD risk score system has its limitation (6). Several laboratory tests, including D-dimer, matrix metalloproteinases (MMPs), and genetic markers, have been developed as biomarkers for the diagnosis of AD, but there still exists some limitations of these biomarkers in the diagnosis of $\mathrm{AD}(7,8)$. Therefore, development of a predictive model that incorporates clinical characteristics and available bedside laboratory tests is desirable. As an individualized and evidence-based predictive model, nomogram is easy to use and can provide accurate information for decision making (9).

The purpose of this study is to develop and validate a nomogram to differentiate between AD and NSTE-ACS in ED. We present the following article in accordance with the TRIPOD Checklist (available at http://dx.doi. org/10.21037/cdt-20-935).

\section{Methods}

The study was conducted in accordance with the Declaration of Helsinki (as revised in 2013). The study was approved by the institutional ethics committee board of Zhenjiang First People's Hospital (No. K-20170032-W) and individual consent for this retrospective analysis was waived.

\section{Patients}

From January 2016 to July 2018, a retrospective study was conducted on 263 patients diagnosed with AD or NSTEACS in the Chest Pain Center of Zhenjiang First People's Hospital. Assuming the nomogram had a sensitivity of $90 \%$ and a specificity of $90 \%$ for differentiating between AD and NSTE-ACS, at least 158 patients should be enrolled. The diagnosis of $\mathrm{AD}$ was based on the result of aortic CTA or MRA. The diagnosis of NSTE-ACS was according to the criteria in the guideline, which included chest pain, ECG and cardiac biomarkers (10). All the diagnoses of NSTE-ACS were confirmed by the coronary artery angiography (CAG). Exclusion criteria included: (I) history of anticoagulation therapy; (II) concomitant with severe infection, shock, disseminated intravascular coagulation, severe renal or hepatic dysfunction, malignancy or other diseases; (III) traumatic AD; (IV) patients who rejected CTA, MRA, CAG or other tests; (V) patients with ST segment elevation on ECG; and (VI) patients < 18 years old.

\section{Collection of data}

All patients were evaluated by the elementary clinical characteristics, ECG and laboratory tests available in ED. Elementary clinical information included sex, age, history of hypertension, diabetes, smoking, history of AD and coronary artery disease (CAD). Chest pain was evaluated by the severity of pain, the quality of pain (tearing pain or not) and the duration of pain. The severity of chest pain was assessed by the Visual Analogue Scale (VAS) (11). In our study, patients with 1-3 scores were defined as mild pain, patients with 4-10 scores were defined as severe pain. Bedside laboratory tests included white blood cell (WBC), platelet (PLT), hemoglobin (Hb), blood urea nitrogen (BUN), creatine (Cr), uric acid (UA), D-dimer and troponin I (TnI). The results of aortic CTA or MRA were also evaluated in patients with $\mathrm{AD}$, and the results of CAG were evaluated in patients with NSTE-ACS.

\section{Statistical analysis}

For continuous variables, the Kolmogorov-Smirnov test was 
used to investigate the distribution of the data. Variables with normal distribution are presented as mean $\pm \mathrm{SD}$, and the Student's $t$-test was used to investigate differences of the variables between the groups. Variables that are not normally distributed are presented with interquartile range and median, and the Mann-Whitney test was used. Percentages were used to summarize the categorial data, and the $\chi^{2}$ or Fisher exact test was used when appropriate to evaluate the differences between groups.

The initial population was randomized to training and validation groups at a $7: 3$ ratio, which were utilized to develop and authenticate the model, respectively. The least absolute shrinkage and selection operator (LASSO) logistic regression model was used to select the factors with significant value of predicting the diagnosis of $\mathrm{AD}$ from the data in the training group. Factors with nonzero coefficients in the LASSO regression model were selected. Then multivariable logistic regression analysis based on the factors selected in the LASSO regression and some other factors with clinical significance, was performed to build a predicting model. Variables with the two-sided $\mathrm{P}$ value $\leq 0.1$ were enrolled in the predictive model, whereas factors with clinical significance were included directly. To predict the probability of $\mathrm{AD}$ quantitatively for an individual patient, we built a nomogram based on the multivariable logistic regression analysis in the training group.

To assess the differentiating value of the nomogram, the area under the curve (AUC) of receiver operating characteristic (ROC) curve and the calibration curve were performed in the training group. Both methods were also used in the validation group to further validate the performance of the nomogram. In addition, decision curve analyses (DCA) were conducted to determine the clinical utility of the nomogram by quantifying the net benefits in both groups. All statistical analyses were performed with SPSS Statistics for Windows, v26.0 (SPSS Inc., USA) and R software (Version 4.0.2; https://www.r-project.org).

\section{Results}

\section{Patients}

A total of 263 patients (94 AD patients and 169 NSTE-ACS patients) were enrolled, of which $70 \%$ were indiscriminately allocated to the training group $(\mathrm{n}=187)$, and the remaining patients were allocated to the validation group $(n=76)$.
Table 1 summarizes clinical characteristics, ECG and laboratory tests between the training group and validation group. Both groups had similar proportion of $\mathrm{AD}$ patients (36.4\% in the training group and $34.2 \%$ in the validation group). Patients with AD in both groups had higher levels of D-dimer, WBC, higher proportion of pulse asymmetry and tearing pain. However, most clinical characteristics and laboratory tests were well-balanced between the training group and validation group.

\section{Feature selection}

Of elementary clinical characteristics, ECG and laboratory tests, 4 out of 20 features were strongly implied as potential predictors of diagnosis of $\mathrm{AD}$ in the training group with 187 patients (Figure 1), which were identified in the LASSO logistic regression model. The features included pain severity, tearing pain, D-dimer level and TnI level. For the usefulness of ECG and pulse asymmetry in clinical practice, these two features were also enrolled (Table 2).

\section{Nomogram construction}

Based on the predictive features identified in the training group, a nomogram that included 4 significant predictors and 2 factors with clinical significance was established for predicting the probability of $\mathrm{AD}$ in this population (Figure 2). Every factor in the nomogram got an individual score according to the value of factor, and a total score was obtained by summarizing the scores of the six factors, which could be used to estimate the probability of AD.

\section{Performance of the nomogram}

Firstly, we validated the effects of the nomogram to differentiate between $\mathrm{AD}$ and NSTE-ACS in the training group. The AUC of the nomogram to predict the diagnosis of $\mathrm{AD}$ was 0.919 (95\% CI, 0.876-0.962) (Figure $3 A$ ). The calibration curve showed that predictive nomogram for the probability of $\mathrm{AD}$ excellently estimated actual probabilities (Figure 3B). Next, the performance of the nomogram was validated by comparing the predictive probability of $\mathrm{AD}$ and the actual clinical results in the validation group. The AUC of the predictive nomogram was 0.938 (95\% CI, 0.888-0.989) (Figure 3C). The calibration curve showed a good consistency between the actual diagnosis of $\mathrm{AD}$ and the predicted probability (Figure 3D). 
Table 1 Baseline characteristics of patients in training and validation groups

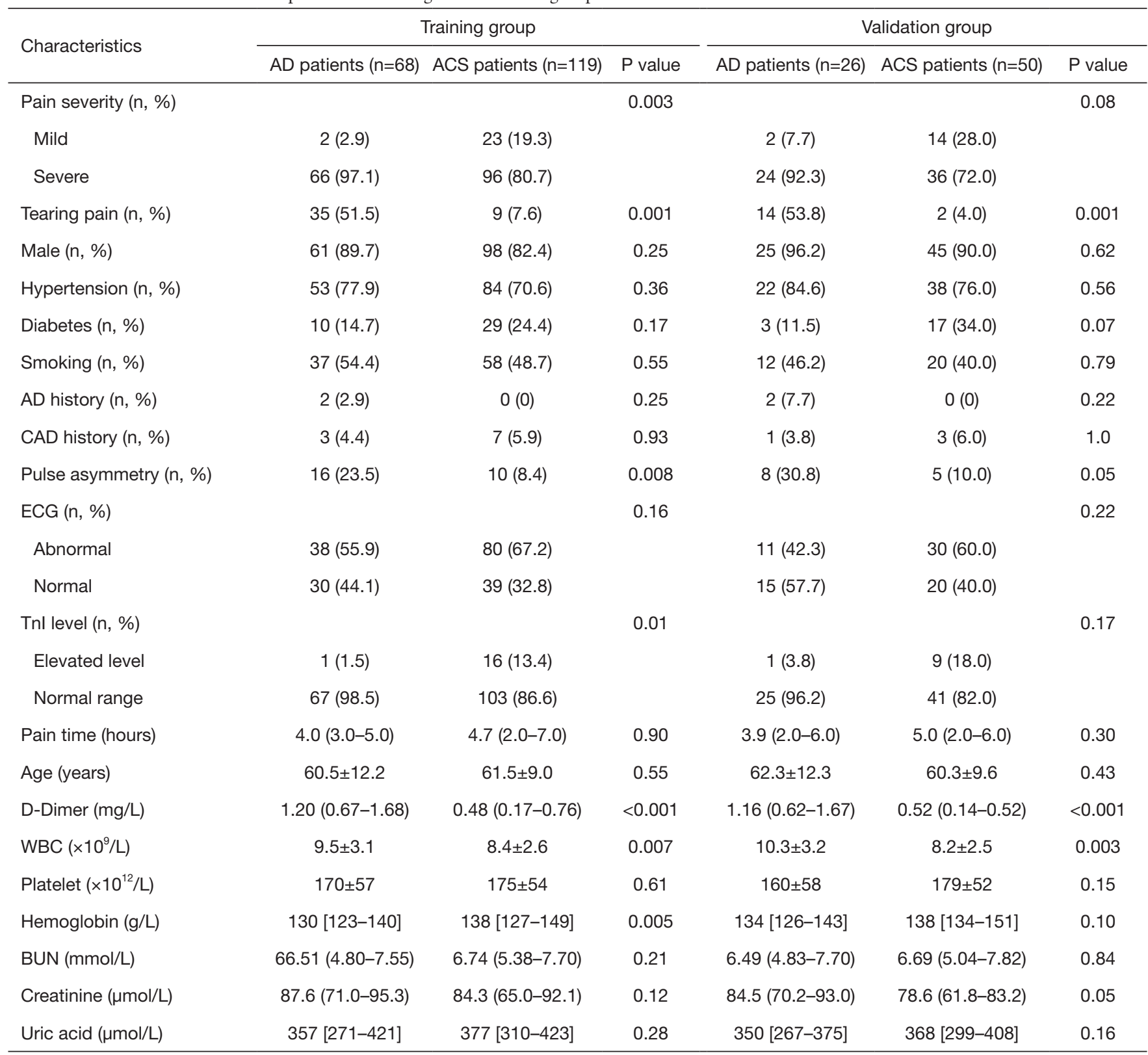

$A D$, aortic dissection; ACS, acute coronary syndrome; CAD, coronary artery disease; ECG, electrography; Tnl, troponin I; WBC, white blood cell; PLT, platelet; BUN, blood urea nitrogen.

\section{Clinical use of the nomogram}

DCA for the nomogram to predict the probability of AD was performed to make sure whether this model can provide great net benefit. The results of DCA showed that the prediction nomogram had excellent overall net benefits in predicting $\mathrm{AD}$ in both the training group and the validation group (Figure $4 A, B$ ).

\section{Discussion}

In this study, we developed a nomogram to differentiate between AD and NSTE-ACS in ED. All features enrolled in our nomogram were elementary clinical information and bedside laboratory tests available, allowing for routine accessibility in clinical practice. In addition, we validated the performance of the nomogram in both the training 

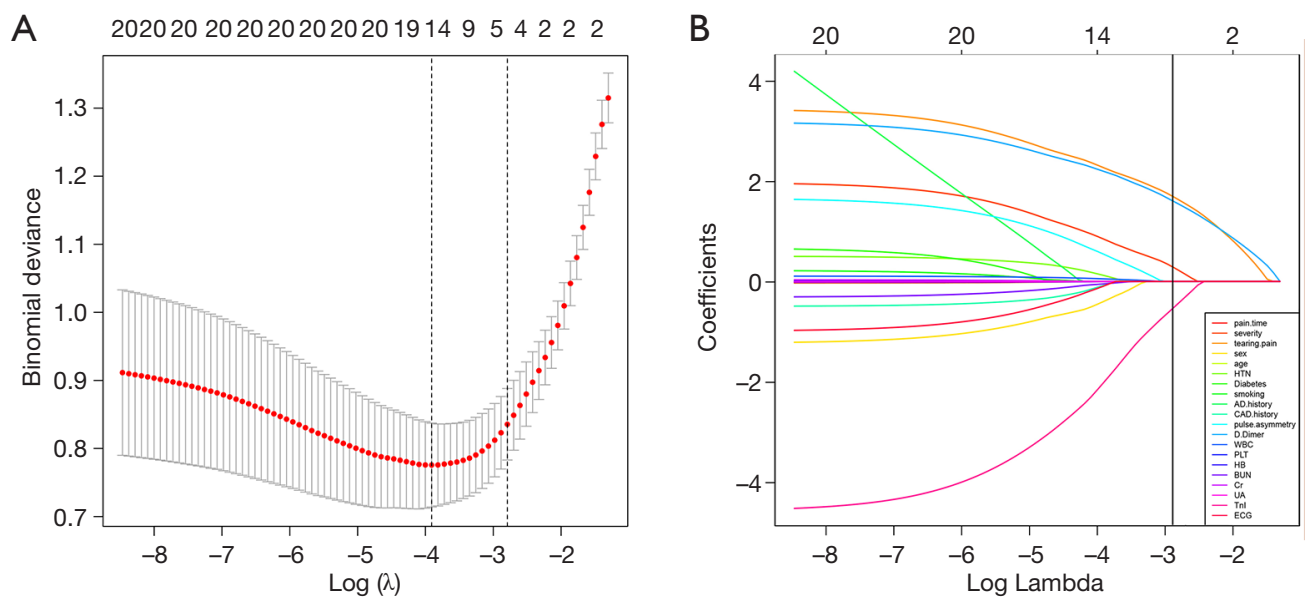

Figure 1 Feature selection by the LASSO logistic regression model. (A) The optimal $\lambda$ selection in the LASSO model with 10 -fold crossvalidation by the minimum criteria (the left dotted vertical line) and the 1-SE criteria (the left dotted vertical line). (B) LASSO coefficient profiles of all the 20 features. Four features with nonzero coefficients were selected by the optimal $\lambda$ where the vertical line was drawn. LASSO, least absolute shrinkage and selection operator; SE, standard error.

Table 2 Predictors for the diagnosis of AD

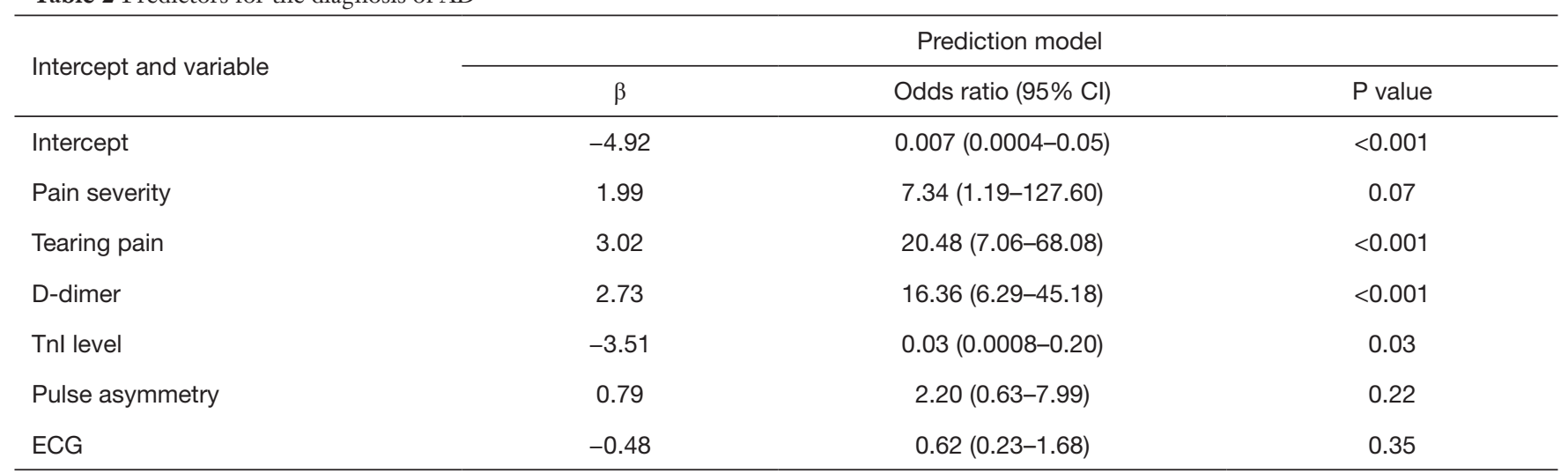

$\mathrm{AD}$, aortic dissection; $\mathrm{Cl}$, confidence interval; Tnl, troponin I; ECG, electrogram.

group and the validation group. The results showed that the nomogram had a good discrimination and calibration in both groups, with an AUC of 0.919 in the training group and 0.938 in the validation group.

In clinical practice, most patients with acute chest pain in ED have to be differentiated from AD, ACS and pulmonary embolism (PE). For the limit overlap between $\mathrm{PE}$ and the other two diseases, $\mathrm{PE}$ can be ruled-in or ruledout easily based on the patients' clinical characteristics (12). However, patients with $\mathrm{AD}$ often have similar symptoms and ECG changes for patients with ACS, especially in patients with non-ST segment elevation on ECG (13), making it challenging for clinicians to rapidly and accurately differentiate between AD and NSTE-ACS. Aortic CTA plays a central role in the diagnosis of $\mathrm{AD}$ presently. However, CTA would expose patients to the risks of radiation, contrast-induced nephropathy and allergies (4). Therefore, helping clinicians reduce the risk of both misdiagnosis and over-testing in differentiation might be of great significance.

Based on the risk factors, pain characteristics and physical examination findings, the ADD risk score was calculated to assess the probability of $\mathrm{AD}$ and recommended in the guidelines for $\mathrm{AD}$. The $\mathrm{ADD}$ risk score ranged from $0-3$ according to the number of variables in which at least one feature was present. Patients with ADD risk score $\geq 2$ 

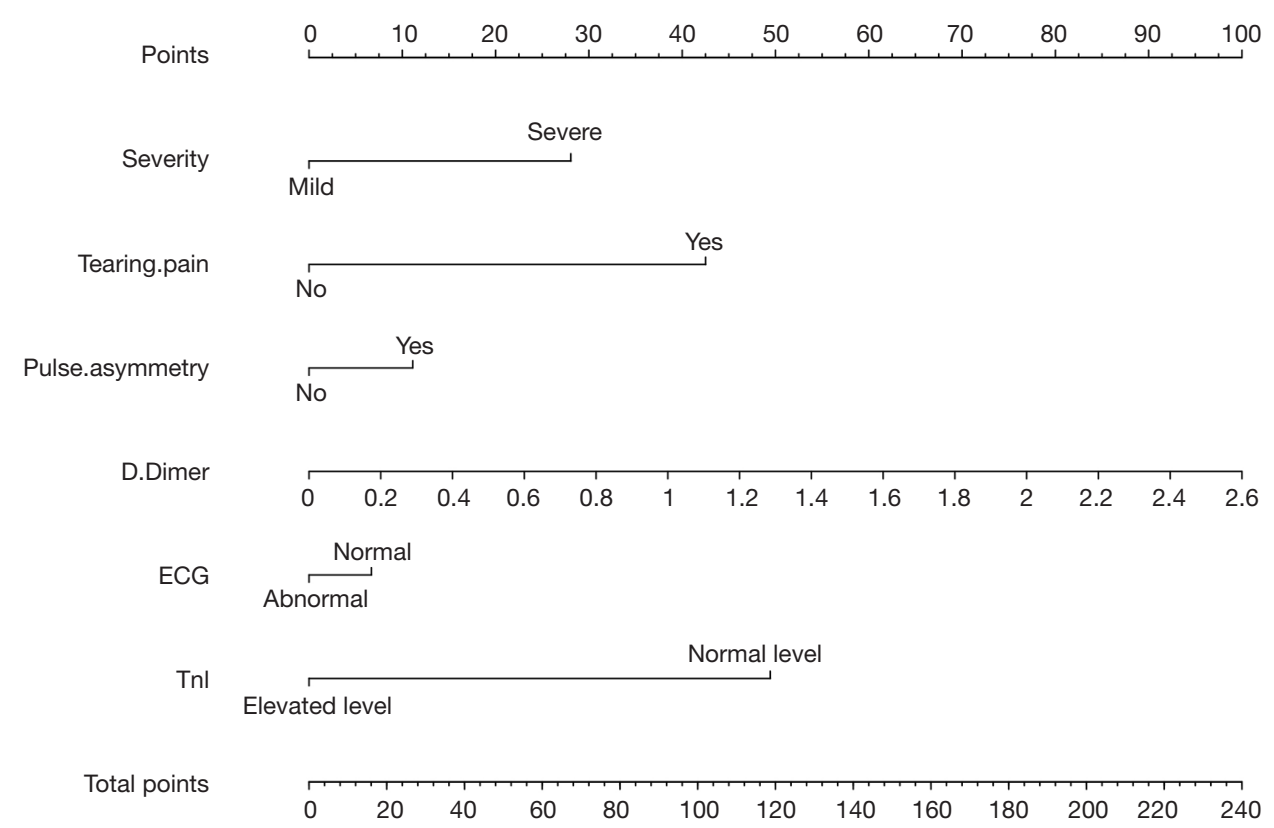

Risk of $A D$

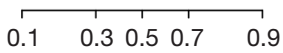

Figure 2 Developed aortic dissection predictive nomogram. The nomogram was developed in the training group, with pain severity, tearing pain, pulse asymmetry, D-dimer levels, ECG and TnI levels incorporated. ECG, electrography; TnI, troponin I.

were suggested to be further assessed by CTA (14), but for patients with ADD risk score $<2$, which might be a large proportion of patients with chest pain, the value of ADD risk score was a limitation $(15,16)$. In addition, the symptoms and physical findings varied among different patients (17). All these factors indicated that a risk score system only based on the elementary clinical conditions was insufficient to rule-in or rule-out $\mathrm{AD}$ patients, especially in the population of $\mathrm{AD}$ and NSTE-ACS.

D-dimer is a thrombotic/fibrinolytic product and available bedside in the ED worldwide. Elevated D-dimer level is as an indicator of the endogenous fibrinolytic activity naturally counteracting the activation of the extrinsic pathway of the coagulation cascade (18). In patients with $\mathrm{AD}$, the rupture of the intima of the aorta permits the blood to contact with the non-endothelialized tissue, which would activate the extrinsic pathway of the coagulation cascade and subsequent endogenous fibrinolytic system (19). Therefore, D-dimer levels is often elevated in patients with AD. Many studies have also shown the good performance of $\mathrm{D}$-dimer in differentiating $\mathrm{AD}$ from other chest pain diseases (20-22). However, elevated D-dimer levels were also found in patients with NSTE-ACS due to the plaque instability in the coronary artery and subsequent activation of the extrinsic coagulation system and the endogenous fibrinolytic system (23). Although the levels of D-dimer were lower in patients of NSTE-ACS than in patients with $\mathrm{AD}$, the predictive value of $\mathrm{D}$-dimer was decreased in the population (24). In addition, normal range D-dimer was also found in patients with $\mathrm{AD}(25)$. All these factors would limit the value of D-dimer in differentiating AD from NSTE-ACS. Troponin is an effective tool in the diagnosis of NSTE-ACS, especially in patients with myocardial infarction. However, owing to the delayed rise in measurable cardiac troponin from the onset of symptoms, the value of conventional troponin is limited for early detection of NSTE-ACS. High-sensitivity troponin can be detected within 1 hour after the onset of chest pain, but the low specificity and availability limits its value in clinical practice (26).

As suggested above, all these tools mentioned are of value in differentiating $\mathrm{AD}$ from other diseases in patients with acute chest pain. However, as they only partially reflect certain aspects of $\mathrm{AD}$, these tools have their limitations. In our study, the multivariable logistic regression analysis showed pain severity, tearing pain, D-dimer and TnI levels 

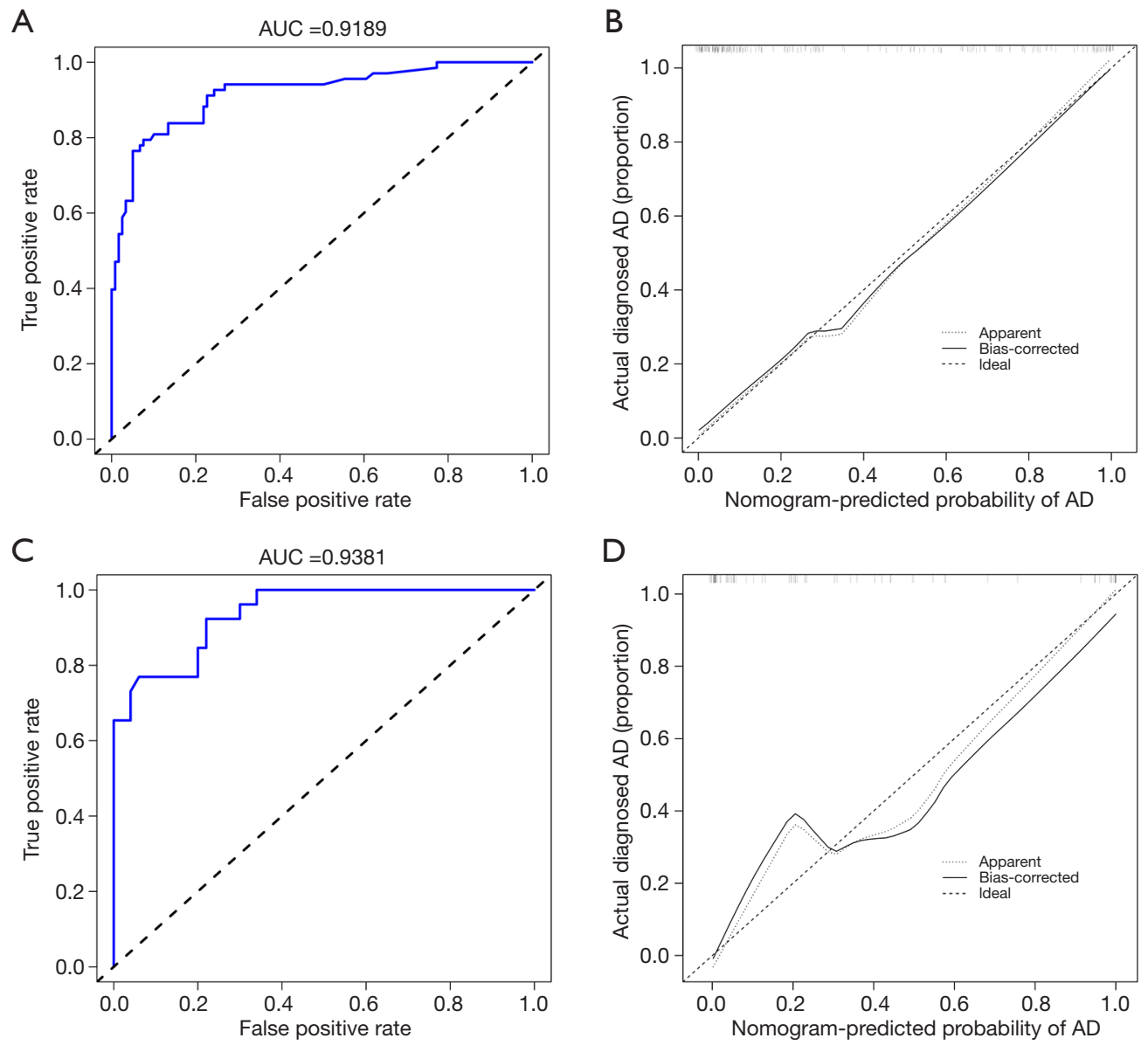

Figure 3 Validation of the nomogram in both the training and validation groups. (A) ROC curve of the nomogram for the prediction of AD in the training group. The AUC was 0.919 (95\% CI, 0.876-0.962). (B) Calibration curve of the nomogram in the training group. (C) ROC curve of the nomogram for the prediction of AD in the validation group. The AUC was 0.938 (95\% CI, 0.888-0.989). (D) Calibration curve of the nomogram in the validation group. ROC, receiver operating characteristic curve; AD, aortic dissection; AUC, area under the curve; $\mathrm{CI}$, confidence interval.

were independent predictors for $\mathrm{AD}$. Based on the values in clinical practice, ECG and pulse asymmetry were also enrolled our nomogram model.

As an accurate and distinguishable predictive tool, nomogram is widely applied in various professional fields to assist modern medical decisions $(27,28)$. Based on the features selected, the nomogram developed to predict the probability of $\mathrm{AD}$ can be served as a viable scoring system. The different lines per predictor in the nomogram represents its relative importance for the prediction, thereby making the interpretation of the results easy to follow. For a clinician struggling to differentiate AD from NSTE-ACS, the probability of $\mathrm{AD}$ can be calculated by combining the scores of each factor on the nomogram (29). The calibration plots showed perfect calibration capacity of the predictive nomogram model for both internal and external validation. All these results indicated that our predictive nomogram had excellent discriminative power and accuracy to differentiate $\mathrm{AD}$ from NSTE-ACS in the population. The DCA in our study showed that the predictive nomogram had higher overall net benefits, and confirmed that the nomogram had the ability to differentiate patients with a high-risk probability of $\mathrm{AD}$.

In addition to the accurate prediction, easy-to-use capability is another important advantage of the nomogram developed in our study. There were only 6 factors in our nomogram, which included symptoms (pain severity and tearing pain), vital sign (pulse asymmetry), ECG and 

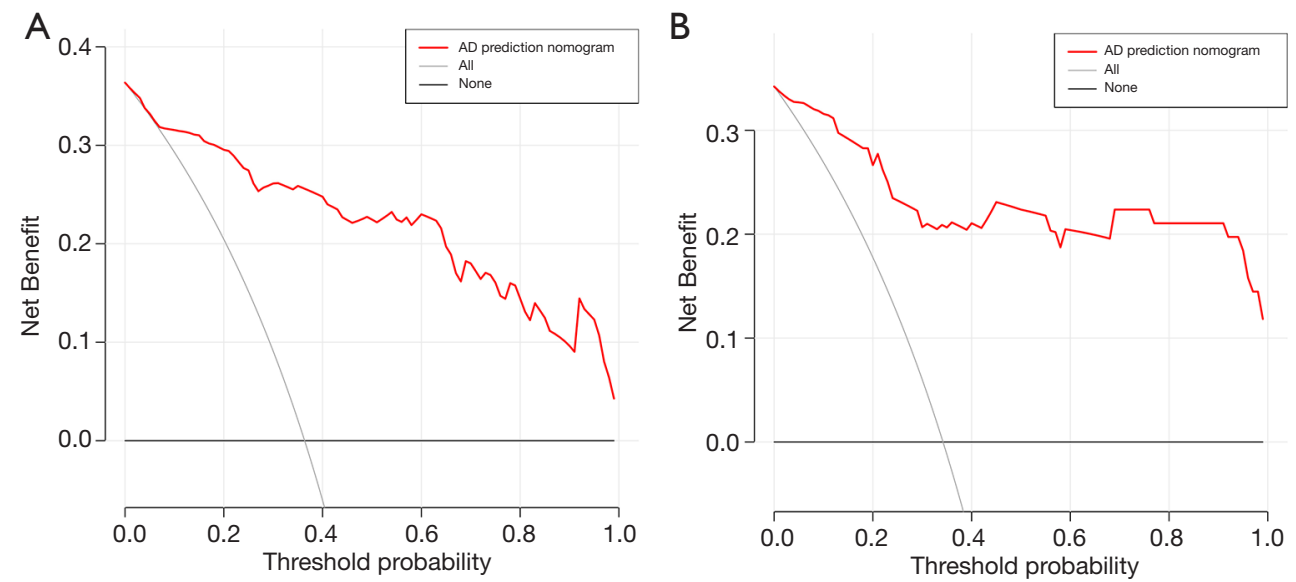

Figure 4 DCA for the nomogram in both the training group (A) and the validation (B) group. The red-line indicates the predictive nomogram. The gray line indicates the assumption that all patients with diagnosis of $\mathrm{AD}$, and black line represent the assumption that no patient with diagnosis of AD. The DCA indicated that the predictive nomogram had higher overall net benefits in predicting AD in both the training group and the validation group. $\mathrm{AD}$, aortic dissection; DCA, decision curve analysis.

laboratory tests (TnI, D-dimer). All these factors can be obtained easily in the ED, which is of great significance for patients with acute chest pain, especially for the high-risk patients who need urgent intervention.

\section{Limitations}

There are several limitations of our study. Firstly, this analysis was based on data from a single center. Although we validated the efficacy of the nomogram in both the training group and the validation group, validation in other centers and with a larger sample size is needed to further assess efficacy. Secondly, for the relatively small sample size and low incidence of risk factors for $\mathrm{AD}$, such as Marfan syndrome and family history, we did not consider these factors in our study. In addition, as a retrospective design, our study has some disadvantages compared to a prospective study. Therefore, further prospective validation of the nomogram to assess its efficacy should be encouraged. Lastly, the patients with cardiac arrest or profound shock who could not undergo CTA or MRA were not enrolled in our study, meaning the nomogram had limited value for these patients.

\section{Conclusions}

In conclusion, we developed and validated a predictive nomogram to differentiate AD from NSTE-ACS based on elementary clinical characteristics, ECG and bedside laboratory tests available. This nomogram can be used as a useful tool to differentiate AD from NSTE-ACS rapidly and accurately in the ED.

\section{Acknowledgments}

We thank all of our colleagues working in the Chest Pain Center, the Affiliated People's Hospital of Jiangsu University. We would like to thank Dr. Tony Smith for his help in polishing our paper.

Funding: None.

\section{Footnote}

Reporting Checklist: The authors have completed the TRIPOD Checklist. Available at http://dx.doi. org/10.21037/cdt-20-935

Data Sharing Statement: Available at http://dx.doi. org/10.21037/cdt-20-935

Conflicts of Interest: All authors have completed the ICMJE uniform disclosure form (available at http://dx.doi. org/10.21037/cdt-20-935). The authors have no conflicts of interest to declare.

Ethical Statement: The authors are accountable for all aspects of the work in ensuring that questions related to the accuracy or integrity of any part of the work are 
appropriately investigated and resolved. The study was conducted in accordance with the Declaration of Helsinki (as revised in 2013). The study was approved by institutional ethics committee board of Zhenjiang First People's Hospital (No. K-20170032-W) and individual consent for this retrospective analysis was waived.

Open Access Statement: This is an Open Access article distributed in accordance with the Creative Commons Attribution-NonCommercial-NoDerivs 4.0 International License (CC BY-NC-ND 4.0), which permits the noncommercial replication and distribution of the article with the strict proviso that no changes or edits are made and the original work is properly cited (including links to both the formal publication through the relevant DOI and the license). See: https://creativecommons.org/licenses/by-nc-nd/4.0/.

\section{References}

1. Bautz B, Schneider JI. High-Risk Chief Complaints I: Chest Pain-The Big Three (an Update). Emerg Med Clin North Am 2020;38:453-98.

2. Xue $\mathrm{Y}$, Chong $\mathrm{H}, \mathrm{Zhu} \mathrm{X}$, et al. Aortic dissection patients mimic acute coronary syndrome with preoperative antiplatelet therapy. J Thorac Dis 2019;11:3385-90.

3. Dudzinski DM, Isselbacher EM. Diagnosis and Management of Thoracic Aortic Disease. Curr Cardiol Rep 2015;17:106.

4. Morello F, Santoro M, Fargion AT, et al. Diagnosis and management of acute aortic syndromes in the emergency department. Intern Emerg Med 2021;16:171-81.

5. Hiratzka LF, Bakris GL, Beckman JA, et al. 2010 ACCF/AHA/AATS/ACR/ASA/SCA/SCAI/SIR/STS/ SVM guidelines for the diagnosis and management of patients with Thoracic Aortic Disease: a report of the American College of Cardiology Foundation/American Heart Association Task Force on Practice Guidelines, American Association for Thoracic Surgery, American College of Radiology, American Stroke Association, Society of Cardiovascular Anesthesiologists, Society for Cardiovascular Angiography and Interventions, Society of Interventional Radiology, Society of Thoracic Surgeons, and Society for Vascular Medicine. Circulation 2010;121:e266-369.

6. Nazerian P, Giachino F, Vanni S, et al. Diagnostic performance of the aortic dissection detection risk score in patients with suspected acute aortic dissection. Eur Heart J Acute Cardiovasc Care 2014;3:373-81.
7. Balmforth D, Harky A, Adams B, et al. Is there a role for biomarkers in thoracic aortic aneurysm disease? Gen Thorac Cardiovasc Surg 2019;67:12-9.

8. Hahne K, Lebiedz P, Breuckmann F. Impact of d-Dimers on the Differential Diagnosis of Acute Chest Pain: Current Aspects Besides the Widely Known. Clin Med Insights Cardiol 2014;8:1-4.

9. Lei Z, Li J, Wu D, et al. Nomogram for Preoperative Estimation of Microvascular Invasion Risk in Hepatitis B Virus-Related Hepatocellular Carcinoma Within the Milan Criteria. JAMA Surg 2016;151:356-63.

10. Roffi M, Patrono C, Collet JP, et al. 2015 ESC Guidelines for the management of acute coronary syndromes in patients presenting without persistent ST-segment elevation: Task Force for the Management of Acute Coronary Syndromes in Patients Presenting without Persistent ST-Segment Elevation of the European Society of Cardiology (ESC). Eur Heart J 2016;37:267-315.

11. Williamson A, Hoggart B. Pain: a review of three commonly used pain rating scales. J Clin Nurs 2005;14:798-804.

12. Qahtani SA, Kandeel AY, Breault S, et al. Prevalence of Acute Coronary Syndrome in Patients Suspected for Pulmonary Embolism or Acute Aortic Syndrome: Rationale for the Triple Rule-Out Concept. J Clin Med Res 2015;7:627-31.

13. Hazui H, Fukumoto H, Negoro N, et al. Simple and useful tests for discriminating between acute aortic dissection of the ascending aorta and acute myocardial infarction in the emergency setting. Circ J 2005;69:677-82.

14. Erbel R, Aboyans V, Boileau C, et al. 2014 ESC Guidelines on the diagnosis and treatment of aortic diseases:

Document covering acute and chronic aortic diseases of the thoracic and abdominal aorta of the adult. The Task Force for the Diagnosis and Treatment of Aortic Diseases of the European Society of Cardiology (ESC). Eur Heart J 2014;35:2873-926.

15. American College of Emergency Physicians Clinical Policies Subcommittee (Writing Committee) on Thoracic Aortic Dissection, Diercks DB, Promes SB, et al. Clinical policy: critical issues in the evaluation and management of adult patients with suspected acute nontraumatic thoracic aortic dissection. Ann Emerg Med 2015;65:32-42.e12.

16. Rawicki AJ, Klim S, Kelly AM. What is the distribution of Aortic Dissection Detection Risk Score in an undifferentiated emergency department chest pain population? Emerg Med Australas 2020;32:872-4.

17. Tsai TT, Nienaber CA, Eagle KA. Acute aortic syndromes. 
Circulation 2005;112:3802-13.

18. Sodeck G, Domanovits H, Schillinger M, et al. D-dimer in ruling out acute aortic dissection: a systematic review and prospective cohort study. Eur Heart J 2007;28:3067-75.

19. Liu Y, Han L, Li J, et al. Consumption coagulopathy in acute aortic dissection: principles of management. J Cardiothorac Surg 2017;12:50.

20. Li W, Huang B, Tian L, et al. Admission D-dimer testing for differentiating acute aortic dissection from other causes of acute chest pain. Arch Med Sci 2017;13:591-6.

21. Gorla R, Erbel R, Kahlert P, et al. Diagnostic role and prognostic implications of D-dimer in different classes of acute aortic syndromes. Eur Heart J Acute Cardiovasc Care 2017;6:379-88.

22. Nazerian P, Mueller C, Soeiro AM, et al. Diagnostic Accuracy of the Aortic Dissection Detection Risk Score Plus D-Dimer for Acute Aortic Syndromes: The ADvISED Prospective Multicenter Study. Circulation 2018;137:250-8.

23. Siennicka A, Jastrzebska M, Smialkowska K, et al. Gender differences in hemostatic and inflammatory factors in patients with acute coronary syndromes: a pilot study. J Physiol Pharmacol 2018;69:91-8.

24. Suzuki T, Distante A, Zizza A, et al. Diagnosis of acute

Cite this article as: Zhang B, Wang Y, Guo J, Zhang G, Yang B. Nomogram to differentiate between aortic dissection and nonST segment elevation acute coronary syndrome: a retrospective cohort study. Cardiovasc Diagn Ther 2021;11(2):457-466. doi: 10.21037/cdt-20-935 aortic dissection by D-dimer: the International Registry of Acute Aortic Dissection Substudy on Biomarkers (IRADBio) experience. Circulation 2009;119:2702-7.

25. Nitta K, Imamura H, Kashima Y, et al. Impact of a negative D-dimer result on the initial assessment of acute aortic dissection. Int J Cardiol 2018;258:232-6.

26. Yang S, Manjunath L, Willemink MJ, et al. The role of coronary CT angiography for acute chest pain in the era of high-sensitivity troponins. J Cardiovasc Comput Tomogr 2019;13:267-73.

27. Yang M, Tao L, An H, et al. A novel nomogram to predict all-cause readmission or death risk in Chinese elderly patients with heart failure. ESC Heart Fail 2020;7:1015-24.

28. Tang XR, Li YQ, Liang SB, et al. Development and validation of a gene expression-based signature to predict distant metastasis in locoregionally advanced nasopharyngeal carcinoma: a retrospective, multicentre, cohort study. Lancet Oncol 2018;19:382-93.

29. Bertens LC, Moons KG, Rutten FH, et al. A nomogram was developed to enhance the use of multinomial logistic regression modeling in diagnostic research. J Clin Epidemiol 2016;71:51-7. 